\title{
Structure of Primordial Gravitational Waves Spectrum in a Double Inflationary Model
}

\author{
David Polarski ${ }^{1,2}$ and Alexei A. Starobinsky ${ }^{a, b}$ \\ ${ }^{1}$ Laboratoire de Modèles de Physique Mathématique, EP93 CNRS \\ Université de Tours, Parc de Grandmont, F-37200 Tours (France) \\ ${ }^{2}$ Département d'Astrophysique Relativiste et de Cosmologie, \\ Observatoire de Paris-Meudon, 92195 Meudon cedex (France) \\ ${ }^{a}$ Landau Institute for Theoretical Physics, \\ Kosygina St. 2, Moscow 117334 (Russia) \\ ${ }^{b}$ Yukawa Inst. for Theor. Physics, Kyoto University, Uji 611 (Japan)
}

October 16, 2018

\begin{abstract}
The gravitational waves (GW) background generated in a double inflationary model, with two scalar fields mutually interacting through gravity only, and its relative contribution $T / S$ to large-angle temperature fluctuations of the relic microwave background are investigated in detail. The relation between $T / S$ and the slope of the GW spectrum $n_{T}$ is shown to be a discriminative test between a slow-roll inflation driven by one scalar field and more complicated models. It is found that the GW amplitude is not exactly zero in minima of spectral oscillations, this property being an observational, in principle, manifestation of GW being in a squeezed vacuum state during inflation.
\end{abstract}

PACS Numbers: 04.62.+v, 98.80.Cq 
Cosmological models in which the early Universe passed through two successive de Sitter (inflationary) phases of quasi-exponential expansion ([1]-[8] and other papers) are usually called double inflationary ones. The initial spectrum of adiabatic perturbations in these models is not approximately flat (i.e., $n \approx 1$, or HarrisonZeldovich like), it has a characteristic scale and more power on larger scales. This represents one of the possible ways to reconcile the cold dark matter model (CDM) with observations of large-scale spatial distributions of galaxies and their peculiar velocities [9] and large-angle fluctuations of the relic microwave background temperature [10], because the observational data imply an excess of power in the perturbation spectrum on scales beginning from $(25-50) h^{-1} \mathrm{Mpc}$ up to the present Hubble radius $R_{H}=\mathrm{cH}_{0}^{-1}=3000 h^{-1} \mathrm{Mpc}$ as compared to the CDM model with flat initial spectrum of adiabatic perturbations (the standard CDM model) normalized to produce a correct galaxy-galaxy correlation function and having the biasing parameter $b \sim 2$ on a scale of $8 h^{-1} \mathrm{Mpc}$.

The most logically economic double inflationary models possess only 2 additional free dimensionless parameters as compared to the standard CDM model. Therefore, they still retain most of the predictive power of the inflationary scenario. Detailed comparison of a number of double inflationary models with recent observational data [11, 12] shows that they are compatible with observations (though for a rather small window of parameters). In [8], we performed a detailed investigation of a simple double inflationary model with two massive scalar fields and found initial spectra of adiabatic perturbations and gravitational waves generated from vacuum quantum fluctuations during a double inflationary stage analytically in the case when there is a pronounced intermediate stage between two stages of inflation. As explained in [12], power spectra for which the relative height of the two "plateaus" in the spectrum of adiabatic perturbations is compatible with observations correspond to values of $\frac{m_{h}}{m_{l}} \approx 10-15$, the latter number increases to 18 if the recently proposed fit to the COBE data corresponding to $Q_{r m s-P S}=20 \mu \mathrm{K}$ for $n=1$ [13] is used (this is only a little bit less than 15-25 that was proposed in [8] before the announcement of the COBE measurements). The intermediate stage between the two inflationary stages is not pronounced in that case. Therefore we have to resort to numerical simulations in order to get the perturbation spectra with good accuracy. A unique and remarkable property of some variants of the inflationary scenario (in particular the chaotic and power-law inflations) is that such a large primordial gravitational wave background is generated from vacuum quantum fluctuations that it is fairly reasonable that a modest, but still significant part of the large-angle fluctuations of the cosmic microwave background (CMB) temperature $\frac{\Delta T(\theta, \varphi)}{T}$ observed by COBE [10] is due to these gravitational waves. That is why it is very important to calculate the ratio of the gravitational waves amplitude to that of scalar perturbations and their relative contribution to the temperature anisotropy in the double inflationary scenario.

In this letter, we extend this analytical and numerical study to the spectrum of primordial gravitational waves and their contribution to the large-angle temperature fluctuations of the CMB. Though the ratio of GW amplitude to that of adiabatic perturbations and the corresponding ratio of their contributions to the large-angle 
$\Delta T / T$ fluctuations appear to be rather small in the case under consideration, this study gives a possibility to obtain important basic conclusions about a discriminative observational test between slow-roll inflation driven by one scalar field and more complicated models, in particular double inflation, and about an observable (in principle) feature indicating that GW were in a pure squeezed state for some time during inflation.

Let us give the essentials of our model of double inflation and generation of perturbations in it. The phenomenological Lagrangian density describing matter and gravity during and near the inflationary stage, is given by

$$
L=-\frac{R}{16 \pi G}+\frac{1}{2}\left(\phi_{h, \mu} \phi_{h}^{, \mu}-m_{h}^{2} \phi_{h}^{2}\right)+\frac{1}{2}\left(\phi_{l, \mu} \phi_{l}^{, \mu}-m_{l}^{2} \phi_{l}^{2}\right)
$$

where $\mu=0, . ., 3, c=\hbar=1, m_{h} \gg m_{l}$ and the Landau-Lifshitz sign conventions are used. The space-time metric has the form

$$
d s^{2}=d t^{2}-a^{2}(t) \delta_{i j} d x^{i} d x^{j}, \quad i, j=1,2,3 .
$$

Spatial curvature may always be neglected because it becomes vanishingly small during the first period of inflation driven by the heavy scalar field. The background fields are homogeneous and classical. The perturbed FRW background, in longitudinal gauge, has a metric given by

$$
d s^{2}=(1+2 \Phi) d t^{2}-a^{2}(t)(1-2 \Psi) \delta_{i j} d x^{i} d x^{j} .
$$

The same mechanism that produces quantum fluctuations of the scalar fields will also produce tensor metric perturbations or gravitational waves. For each of the polarization states $\lambda$, where $\lambda=1,2$, and the polarization tensor is normalized by the condition $e_{i j} e^{i j}=2$, the amplitude $h_{\lambda}$ is given by

$$
h_{\lambda}=\sqrt{16 \pi G} \phi_{\lambda}
$$

where $\phi_{\lambda}$ is a massless scalar field satisfying

$$
\square \phi_{\lambda}=0 .
$$

Let us consider the spectrum of tensor and scalar adiabatic perturbations

$$
h(k)=B k^{-3 / 2}, \quad \Phi(k)=\frac{3}{10} A k^{-3 / 2} .
$$

The quantities $A$ and $B$ are those introduced in [14, 15], they are only weakly depending on $k$ while $h(k)$ and $\Phi(k)$ are defined as follows

$$
\begin{aligned}
\left\langle h_{\mathbf{k}, \lambda}, h_{\mathbf{k}^{\prime}, \lambda^{\prime}}^{*}\right\rangle & \equiv \frac{1}{2} h^{2}(k) \delta^{(3)}\left(\mathbf{k}-\mathbf{k}^{\prime}\right) \delta_{\lambda \lambda^{\prime}}, \\
\left\langle\Phi_{\mathbf{k}} \Phi_{\mathbf{k}^{\prime}}^{*}\right\rangle & =\Phi^{2}(k) \delta^{(3)}\left(\mathbf{k}-\mathbf{k}^{\prime}\right)
\end{aligned}
$$


where the Fourier transform conventions are $\Phi_{k} \equiv \frac{1}{(2 \pi)^{\frac{3}{2}}} \int \Phi(\mathbf{r}) e^{-i \mathbf{k r}} d^{3} \mathbf{k}$. We assume also that $a(t) \propto t^{2 / 3}$ today.

Let us first consider the ratio of the amplitudes of GW and adiabatic perturbations generated in double inflation. For wavelengths well outside the Hubble radius, $\frac{k}{a} \ll H$, each Fourier mode $h_{k, \lambda}(\eta)$ has a constant value. If they cross the Hubble radius during a quasi-de Sitter stage, $h(k)$ is given by

$$
h(k)=\sqrt{16 \pi G} \frac{H\left(t_{k}\right)}{\sqrt{k^{3}}}
$$

where $t_{k}$ is the Hubble radius crossing time defined as $\frac{k}{a\left(t_{k}\right)}=H\left(t_{k}\right)$. In (9) both polarization states are taken into account. Note that long wavelengths cross the Hubble radius towards the end of the first inflation, so that $H\left(t_{k}\right)=\sqrt{\frac{2}{3} m_{h}^{2} \ln \frac{k_{b}}{k}}$ in agreement with our numerical simulations

$$
k^{\frac{3}{2}} h(k)=\sqrt{\frac{32 \pi G m_{h}^{2}}{3}} \sqrt{\ln \frac{k_{b}}{k}}=\sqrt{\frac{32 \pi G m_{h}^{2}}{3}} \sqrt{s(k)-s_{0}}, \quad s>s_{0}
$$

where $k_{b}$ is the characteristic scale of the spectrum corresponding to the end of the first inflation driven by $\phi_{h}, s=2 \pi G\left(\phi_{l}^{2}+\phi_{h}^{2}\right)$ is the number of e-folds till the end of the second inflation, $s(k) \equiv s\left(t_{k}\right)=\ln \frac{k_{f}}{k}=\ln \frac{a_{f}}{a}, k_{f}=a_{f} H_{f}$ where the index $f$ denotes the values of all quantities at the end of the second inflation, and $s_{0} \gg 1$ is the total number of e-folds during the second inflation. We take $s_{0}=2 \pi G \phi_{0}^{2} \approx 60$, in order to get the characteristic scale $k_{b}$ at the right place today. For the scalar adiabatic perturbations we have [8]

$$
k^{\frac{3}{2}} \Phi(k)=\frac{6 \sqrt{2} \pi G}{5}\left(H \sqrt{\phi_{l}^{2}+\phi_{h}^{2}}\right)_{t_{k}}=\frac{\sqrt{24 \pi G m_{h}^{2}}}{5} \sqrt{s(k)\left(s(k)-s_{0}\right)}, \quad s>s_{0} .
$$

As mentioned above, we are mostly interested in those values of $\frac{m_{h}}{m_{l}}$ for which the intermediate stage, though it exists, is not well pronounced and deviations from monotonous behaviour of $h(k)$ and $\Phi(k)$ are concentrated in the region $\left|s-s_{0}\right| \sim 1$. If the Hubble radius is crossed during the second inflation, we obtain the usual result for inflation driven by one scalar field

$$
k^{\frac{3}{2}} h(k)=\sqrt{\frac{32 \pi G m_{l}^{2}}{3}} \sqrt{s(k)}, \quad k^{\frac{3}{2}} \Phi(k)=\frac{\sqrt{24 \pi G m_{l}^{2}}}{5} s(k), \quad s<s_{0} .
$$

This finally yields

$$
R(k) \equiv \frac{h(k)}{\Phi(k)}=\frac{5 \sqrt{2}}{3 \sqrt{\pi G}\left(\sqrt{\phi_{l}^{2}+\phi_{h}^{2}}\right)_{t_{k}}}=\frac{10}{3 \sqrt{s(k)}}
$$

for both $s>s_{0}$ and $s<s_{0}$, aside from the region $\left|s-s_{0}\right| \leq s_{0} \frac{m_{l}^{2}}{m_{h}^{2}} \sim 1$ around the point $s=s_{0}$. For $s \approx s_{0}, R(k) \approx 0.43$. The behaviour of $R(k)$ obtained numerically (see Figs.2-3) is in excellent agreement with (13). The slope of $R(k)$ is:

$$
\frac{d \ln R(k)}{d \ln k} \equiv \frac{1}{2}\left(n_{T}-n_{S}+1\right)=\frac{1}{2 s(k)} \ll 1
$$


where $n_{S} \equiv 1+\frac{d \log \left(k^{3} \Phi^{2}(k)\right)}{d \log k}, n_{T} \equiv \frac{d \log \left(k^{3} h^{2}(k)\right)}{d \log k}$. In accordance with (14), we see from Fig. 1 that $h(k)$ has a shape very similar to that of $\Phi(k)$. However, this is not surprising since we have shown numerically [16] that the contribution of the light scalar field perturbations to $\Phi(k)$ is dominant for the values of $\frac{m_{h}}{m_{l}}$ considered here, while on the other hand the equation governing the light scalar field fluctuations essentially coincides with eq. (5), the equation for gravitational waves.

We see that the relation

$$
n_{T} \approx n_{S}-1
$$

(proposed, e.g., in [17]) which has a very narrow region of applicability even for the case of single inflation, namely it is valid for power-law inflation only while it is not valid for chaotic inflation (see also the discusion in [18]), turns out to be valid for double inflation for large scales satisfying $k \ll k_{b} \equiv k_{f} e^{-s_{0}}$ (and not too small), as can be seen from (14) and also from the figures. As shown in [12, the best fit to observational data is given by $2 \pi k_{b}^{-1} \approx(6-10) h^{-1} \mathrm{Mpc}$, so the condition $k \ll$ $k_{b}\left(s(k)>s_{0}\right)$ is certainly satisfied for scales probed by CMB anisotropy experiments on large and intermediate angles. On smaller scales for which $k>k_{b}$, the relation (15) is not valid any more. Hence, it cannot be considered as a test neither for generic inflation nor even for a single inflation. More general is the following relation which is valid for any slow-roll inflation driven by one scalar field:

$$
\begin{array}{r}
\frac{T}{S} \equiv \frac{\left\langle\left|a_{l m}\right|^{2}\right\rangle_{G W}}{\left\langle\left|a_{l m}\right|^{2}\right\rangle_{A P}}=\mathcal{K}_{l}\left(\frac{3}{10} R(k)\right)^{2}=\mathcal{K}_{l}\left|n_{T}\right|, \quad n_{T}<0, \\
\mathcal{K}_{l}=\text { const }=\frac{25}{9}\left(1+\frac{48 \pi^{2}}{385}\right) \simeq 6.20, \quad l \gg 1, \quad\left|n_{T}\right| \ll 1, \\
\mathcal{K}_{2} \approx 6.93, \quad \mathcal{K}_{3} \approx 5.45, \quad \mathcal{K}_{5} \approx 5.10, \quad \mathcal{K}_{10} \approx 5.30, \quad\left|n_{T}\right| \ll 1
\end{array}
$$

with $\mathcal{K}_{5}$ being the minimal value for integer $l<40$. In these expressions, only the Sachs-Wolfe effect is taken into account for both types of perturbations. Eq. (17) was first derived in [15], and (18) follows from results obtained in [19, 15] (see also recent calculations in [20]). However, the quadrupole was not detected by COBE and it is usually excluded from the data; the asymptotic value (17) is not reached either for multipoles $l=3-15$ measured by COBE. Taking into account the Doppler and the Silk effects for adiabatic perturbations yields a further decrease of $\mathcal{K}_{10}$ by $\approx 10 \%$. Thus, it is better to use the value $\mathcal{K}_{l} \sim 5$ when estimating the GW contribution to the actual COBE data.

The relation (16) is strongly violated in the case of double inflation for $s(k)>s_{0}$ because we then have:

$$
\frac{T}{S}=\mathcal{K}_{l}(0.3 R(k))^{2}=\mathcal{K}_{l}(s(k))^{-1}, \quad\left|n_{T}\right|=\left(s(k)-s_{0}\right)^{-1},
$$

therefore, $T / S \ll \mathcal{K}_{l}\left|n_{T}\right|$. Note that relation (17) is not valid either for the $R+R^{2}$ inflationary model [14, 15]. So, (16) represents a decisive test for single inflation driven by a scalar field. Still this test is difficult because it first requires identification of the GW contribution to the $\frac{\Delta T}{T}$ fluctuations. Also, it cannot be used to predict the 
amount of this contribution using the total COBE data since both sides of relation (16) (in contrast to (15)) contain quantities referring to GW.

For $s \approx s_{0}$, we get $T / S=\mathcal{K}_{10} s_{0}^{-1} \approx 0.08$. As contributions to the $\frac{\Delta T}{T}$ fluctuations from gravitational waves and adiabatic perturbations are statistically independent, the total $r m s$ value of $\frac{\Delta T}{T}$ at large angles gets increased by a factor $\sqrt{1+(T / S)}$, i.e. by about $4 \%$, due to the GW background. Therefore, in our model the $\frac{\Delta T}{T}$ anisotropy on scales probed by COBE comes mainly from adiabatic perturbations. However, when comparing predictions of the model with observations [12], the GW contribution has to be taken into account.

Now we consider the dip in the $R(k)$ curve (see Figs.2-4) lying in the transition region between the two "plateaus" that arises for $\frac{m_{h}}{m_{l}} \geq 15$, i.e. when the intermediate period between two phases of inflation is sufficiently pronounced. Though, as can be seen from the figures, it constitutes a small effect even for $\frac{m_{h}}{m_{l}}=25$, it is very interesting theoretically for two reasons. First, its very existence is due to the effect discovered in [8]: the necessity of taking into account a contribution of the heavy scalar field quantum fluctuations to the adiabatic perturbations in this range of scales. Indeed, in the absence of break between two phases of inflation $\left(\frac{m_{h}}{m_{l}}<15\right)$, quantum fluctuations of the light scalar field produce the dominant contribution to adiabatic perturbations over the region involved since $\phi_{l}=\phi_{0} \gg \phi_{h} \sim 0.5 \mathrm{M}_{\mathrm{p}}$. But if there exists a pronounced intermediate power-law stage $\left(s_{0} m_{l}^{2} m_{h}^{-2} \ll 1\right)$, the contribution of the heavy scalar field fluctuations becomes important in the transition region between the upper "plateau" and the lower one. It is given by the second term in the r.h.s. of eq.(4.18) of ref. [8]:

$$
\begin{array}{r}
k^{\frac{3}{2}} \Phi(k)=\frac{3 \sqrt{3 \pi G m_{h}^{2}}}{5 \sqrt{2}} \ln ^{1 / 2} \frac{k_{b}}{k}\left(2 \pi G \phi_{0}^{2}\left(\frac{k_{1}}{k}\right)^{6} \sin ^{2}\left(\sigma \frac{k}{k_{1}}\right)+\left(2 \pi \sigma_{1}\right)^{2}\left(\frac{k_{1}}{k}\right)^{2} \ln \frac{k_{b}}{k}\right)^{\frac{1}{2}}, \\
\sigma_{1} \approx 0.2875, \quad \sigma \approx 2.804, \quad k_{1} \simeq k_{b}\left(\frac{s_{0} m_{l}^{2}}{m_{h}^{2}}\right)^{1 / 6}, \quad k_{1} \lesssim k \lesssim k_{b},
\end{array}
$$

see eq. (4.32) in [8] for $k>k_{b}$. On the other hand (see [21, 8] and Fig.18.7, p.312 in 22]),

$$
k^{3 / 2} h(k)=\sqrt{6 \pi G m_{h}^{2} \ln \left(\frac{k_{b}}{k}\right)}\left(\frac{k_{1}}{k}\right)^{3}\left|\sin \left(\sigma \frac{k}{k_{1}}\right)\right|, \quad k_{1} \lesssim k \lesssim k_{b} .
$$

The first, oscillating, term in the r.h.s. of (20) arises from the light scalar field fluctuations and is proportional to the r.h.s. of (21), while the second does not. In the absence of the heavy scalar field contribution, $R(k)$ would be a smooth function of $k$. But the second term becomes important near the points where $\sin \left(\sigma \frac{k}{k_{1}}\right)=0$, or $k=1.12 k_{1}$ preventing $\Phi(k)$ from going to zero in contrast to $h(k)$. This explains why $R(k)$ has a dip. For the values of $\frac{m_{h}}{m_{l}}$ considered here, this effect is not big and can be calculated quantitatively with numerical methods only. Neverhteless, the appearance of the dip in the $R(k)$ curve clearly shows that the heavy field contribution is present even if definitely subdominant. 
A second new and remarkable point is that the minimum of the dip is not exactly zero as follows from the previously known eq.(21). This is a direct consequence of the GW background being produced from the initial vacuum state during the first de Sitter stage via the process of particle creation, or in other words, of the GW being in a squeezed vacuum quantum state during inflation. The mathematical expression of this fact is that the amplitude $h_{\lambda}(k)$ of the GW (operator) field modes has the following form in the regime $k \ll a H$ :

$$
h_{\lambda}(k)=\sqrt{16 \pi G}\left(C_{1}+C_{2} \int_{\infty}^{t} \frac{d t}{a^{3}(t)}\right), \quad C_{1}=\frac{H\left(t_{k}\right)}{\sqrt{2 k^{3}}}, \quad C_{2}=-\frac{i k^{3 / 2}}{\sqrt{2} H\left(t_{k}\right)} .
$$

The first term in (22) is the quasi-isotropic mode which was made purely real by a time-independent phase rotation, the second one is the decaying mode. The condition $C_{1} \Im C_{2}=-\frac{1}{2}$ expressing quantum coherence between the two modes reflects the fact that initially, for $t \rightarrow-\infty, k \gg a H$, the quantum state of the mode was the vacuum. Both terms in the right-hand side of eq. (22) are of the same order at the Hubble radius crossing during the first inflation. After that, the decaying mode quickly becomes exponentially small. If the latter mode is neglected as is usually done, then quantum coherence is lost and the resulting state of the GW for given $\mathbf{k}$ becomes equivalent to a classical wave with a Gaussian stochastic amplitude, a fixed temporal phase and some special correlation between $\mathbf{k}$ and $\mathbf{- k}$ modes (see the detailed discussion in 223]). Actually, the quantum coherence is most probably lost shortly after the end of the second inflation due to interactions of the decaying mode with the environment though, as explained in [23], there is no necessity to consider these interactions explicitly. As a result, the present GW background is not in a squeezed vacuum quantum state, it is purely classical. However, it is natural to assume that quantum coherence is not lost throughout inflation, at least if the decaying mode is not negligible.

Then, for the double inflationary model, the decaying mode appears to be nonnegligible over some range of $k$ close to $k_{b}$ due to the existence of a break between the two inflationary stages which yields two more Hubble radius crossings for modes belonging to that range. Keeping both terms in (22), in contrast to previous calculations [21, 8], and using the method developed in [8], we obtain the following result for $h_{\lambda}(k)$ after the last Hubble radius crossing during the second inflation (in the limit $\left.s_{0} m_{l}^{2} m_{h}^{-2} \ll 1\right)$ :

$$
\begin{aligned}
h_{\lambda}(k) & =D_{1} \sin \left(\frac{k}{k_{1}} \sigma\right)+i D_{2} \cos \left(\frac{k}{k_{1}} \sigma\right) \\
D_{1} & =-\frac{3}{4}\left(\frac{k_{1}}{k}\right)^{3} \frac{H\left(t_{k}\right)}{\sqrt{2 k^{3}}}, \quad D_{2}=-\frac{2 \sqrt{2} H_{0}^{2} k^{3 / 2}}{3 H\left(t_{k}\right) k_{1}^{3}} .
\end{aligned}
$$

where $H_{0}$ is the Hubble parameter at the beginning of the second inflation. Note that $h_{\lambda}(k)$ could be made purely real had we chosen to make a different time-independent phase rotation.

It is clear that the minimum of the dimensionless quantity $k^{3 / 2} h(k)$, though small, 
is not zero and it is reached at $k \approx \pi \frac{k_{1}}{\sigma}$ :

$$
\begin{aligned}
k^{3 / 2} h_{\min }(k) & =\sqrt{32 \pi G} k^{3 / 2}\left|D_{2}\right|=\frac{16 \pi^{3} \sqrt{\pi G} H_{0}^{2}}{3 \sigma^{3} H\left(t_{k}\right)} \approx \frac{10 m_{l}^{2} s_{0}}{m_{h} \mathrm{M}_{\mathrm{p}}}, \\
\frac{h_{\min }(k)}{h_{\max }(k)} & =\frac{8 \sqrt{2}}{9}\left(\frac{k}{k_{1}}\right)^{6} \frac{H_{0}^{2}}{H^{2}\left(t_{k}\right)}=\frac{8 \sqrt{2} \pi^{6}}{9 \sigma^{6}} \frac{m_{l}^{2} s_{0}}{m_{h}^{2} \ln \left(\frac{\sigma k_{f}}{\pi k_{1}}\right)} \approx 2.5 \frac{s_{0} m_{l}^{2}}{m_{h}^{2}} .
\end{aligned}
$$

Hence, though the mode $h_{\lambda}(k)$ is semiclassical after inflation, we may say that there appears a "quantum signature" in the present spectrum of the GW background due to the fact that the decaying mode should be taken into account in order to derive the correct form of the spectrum.

Eq.(24) is in very good agreement with the numerical simulation displayed on Fig.1, where the dimensionless quantity $k^{3 / 2} h(k)$ is expressed in $m_{h} / \mathrm{M}_{\mathrm{p}}$ units. For $p=$

25 , the value of the minimum $\approx 0.2$ which yields the value $\frac{k}{k_{1}} \approx 1.14$, in remarkable agreement with (24). Keeping the decaying mode in the scalar perturbations too, we get for $R_{\min }$ :

$$
R_{\text {min }}=\frac{10}{3 \sqrt{s_{0}}} \frac{1}{\sqrt{1+\left(\frac{9 \pi \sigma_{1}}{8} \frac{H^{2}\left(t_{k}\right) \phi_{h}\left(t_{k}\right)}{H_{0}^{2} \phi_{0}}\left(\frac{k_{1}}{k}\right)^{4}\right)^{2}}}=\frac{10}{3 \sqrt{s_{0}}} \frac{1}{\sqrt{1+\left(\frac{9 \sigma_{1} \sigma^{4} m_{h}^{2} \ln ^{3 / 2}\left(\frac{k_{1}}{k}\right)}{8 \pi^{3} m_{l}^{2} s_{0}^{3 / 2}}\right)^{2}}}
$$

for the first minimum $k=k_{1} \frac{\pi}{\sigma}$. The value of $\frac{h_{\min }}{h_{\max }}$ and $R_{\min }$ in the higher minima $k=n k_{1} \frac{\pi}{\sigma}, n>1$, quickly grows and the oscillations become unnoticeable.

Thus, the spectrum $h^{2}(k)$ has no zeroes. Note that the necessity of taking into account both modes resulting in the absence of zeroes (despite the existence of deep wells) in the perturbation spectrum may arise even for adiabatic perturbations generated during inflation driven by one scalar field, provided the slow-rolling condition breaks for a short period of time, e.g. near values of the scalar field where the first derivative of its potential experiences a fast change [24]. So, we can formulate the following quantum positivity principle: if perturbations are generated by the process of particle creation from the vacuum, their power spectrum $P(k)$ is non-zero for all modes. Of course, the reverse statement that the absence of zeroes implies that the fluctuations arise from quantum vacuum fluctuations, is not true. But the appearance of zeroes in a power spectrum implies the classical origin of perturbations, and the acoustic (Sakharov) oscillations in a purely baryon-radiation Universe is an example of a classical process leading to a power spectrum with zeroes for some values of $k$.

\section{Acknowledgments}

This work was started when one of the authors (A.S.) was a visiting professor in the Yukawa Institute for Theoretical Physics, Kyoto University, and finished when he was visiting France under the agreement between the Landau Institute for Theoretical Physics and Ecole Normale Supérieure, Paris. A.S. thanks YITP, ENS and EP93 CNRS (Tours) for financial support, Profs. Y. Nagaoka and J. Yokoyama for their hospitality in YITP and Profs. E. Brezin and C. Barrabes for the hospitality in ENS 
and Université de Tours respectively. A.S. was also supported in part by the Russian Foundation for Basic Research, grant 93-02-3631.

\section{References}

[1] L. A. Kofman, A. D. Linde, A. A. Starobinsky, Phys. Lett. B 157, 361 (1985)

[2] A. A. Starobinsky, JETP Lett. 42, 152 (1985)

[3] L. A. Kofman, A. D. Linde, Nucl. Phys. B 282, 555 (1987)

[4] J. Silk, M. S. Turner, Phys. Rev. D 35, 419 (1987)

[5] L. Kofman, D. Pogosyan, Phys. Lett. B 214, 508 (1988)

[6] D. S. Salopek, J. R. Bond, J. M. Bardeen, Phys. Rev. D 40, 1753 (1989)

[7] S. Gottlöber, V. Müller, A. A. Starobinsky, Phys. Rev. D43, 2510 (1991)

[8] D. Polarski, A. A. Starobinsky, Nucl. Phys. B 385, 623 (1992)

[9] S. J. Maddox, G. Efstathiou, W. J. Sutherland, J. Loveday, Mon. Not. Roy. Astron. Soc. 242, 43P (1990); G. Efstathiou et al., Mon. Not. Roy. Astron. Soc. 247, 10P (1990); J. P. Ostriker, Y. Suto, Ap. J. 348, 378 (1990); E. Bertschinger, A. Dekel, S. M. Faber, A. Dressler, D. Burstein, Ap. J. 364, 370 (1990)

[10] G. F. Smoot, C. L. Bennett, A. Kogut, E. L. Wright et al, Ap. J. Lett. 396, L1 (1992); E. L. Wright et al, Ap. J. 396, L13 (1992)

[11] S. Gottlöber, J. P. Mücket, A. A. Starobinsky, Ap. J., 434, 417 (1994)

[12] P. Peter, D. Polarski, A. A. Starobinsky, Phys. Rev. D 50, 4827 (1994)

[13] K. M. Gorski, G. Hinshaw, A. J. Banday et al., Ap. J. 430, L89 (1994); E. F. Bunn, D. Scott, M. White, Ap. J. 441, L9 (1995)

[14] A. A. Starobinsky, Sov. Astron. Lett. 9, 302 (1983)

[15] A. A. Starobinsky, Sov. Astron. Lett. 11, 133 (1985)

[16] D. Polarski, Phys. Rev. D 49, 6319 (1994)

[17] R. Crittenden, J. R. Bond, R. L. Davis, G. Efstathiou, P. J. Steinhardt, Phys. Rev. Lett. 71, 324 (1993); J. R. Bond, R. Crittenden, R. L. Davis, G. Efstathiou, P. J. Steinhardt, Phys. Rev. Lett. 72, 13 (1994)

[18] E. W. Kolb, S. L. Vadas, Phys. Rev. D 50, 2479 (1994)

[19] V. A. Rubakov, M. V. Sazhin, A. V. Veryaskin, Phys. Lett. B 115, 189 (1982); R. Fabbri, M. Pollock, Phys. Lett. B 125, 445 (1983) 
[20] S. Koranda, B. Allen, Phys. Rev. D (1995) astro-ph/9410049).

[21] V. F. Mukhanov, M. I. Zelnikov, Phys. Lett. B 263, 169 (1991)

[22] V. F. Mukhanov, H. A. Feldman, R. H. Brandenberger, Phys. Rep. 215, 203 (1992)

[23] D. Polarski, A. A. Starobinsky, Class. Quantum Grav. (1995), submitted

[24] A. A. Starobinsky, JETP Letters 55, 489 (1992) 
Figure 1: The quantities $\log k^{3 / 2} \Phi(k)$ (upper curve) and $\log k^{3 / 2} h(k)$ (bottom curve), with the dimensionless quantities $k^{3 / 2} \Phi(k)$ and $k^{3 / 2} h(k)$ expressed in $\frac{m_{h}}{M_{\mathrm{p}}}$ units, are displayed as functions of $\log k$ ( $k$ scale is arbitrary) for $\frac{m_{h}}{m_{l}}=25, k \ll a H$. For the upper curve $a(t) \propto t^{2 / 3}$ nowadays is assumed. We note the presence of an oscillation in the transition region. The minimum of $k^{3 / 2} h(k)$ is not zero due to the presence of the decaying mode as explained in the text. We note also the similarity of both curves for the $k$-range displayed here corresponding to wavelengths that cross the Hubble radius towards the end of the first inflation (upper "plateau") and at the beginning of the second inflation (lower plateau).

Figure 2: The quantity $\log R(k) \equiv \log \frac{h(k)}{\Phi(k)}$ is displayed as a function of $\log k$ for $\frac{m_{h}}{m_{l}}=25$. The $k$ scale is the same as in Fig.1. A dip is clearly seen in the transition region due to the contribution of the heavy scalar field fluctuations (see text). Note again that the bottom of the dip is not zero.

Figure 3: The quantity $\log R(k) \equiv \log \frac{h(k)}{\Phi(k)}$ is displayed as a function of $\log k$ for: a) $\frac{m_{h}}{m_{l}}=20$ b) $\frac{m_{h}}{m_{l}}=15$. Again, a dip is seen which decreases with decreasing $\frac{m_{h}}{m_{l}}$, due to the fact that for decreasing values of $\frac{m_{h}}{m_{l}}$, the intermediate stage becomes less pronounced and the contibution of the heavy scalar field fluctuations decreases in the transition region. 
This figure "fig1-1.png" is available in "png" format from: http://arxiv.org/ps/astro-ph/9505125v1 